Artículo

\title{
Ácido salićlico induce tolerancia al estrés por criogenia en Solanum tuberosum
}

\author{
Diana Daniela Ayala-Hernández ${ }^{1}$ \\ Diana Rocío Ruiz-Saénz ${ }^{1}$ \\ Esmeralda Judith Cruz-Gutiérrez ${ }^{2}$ \\ Humberto Antonio López-Delgado ${ }^{1 \S}$ \\ ${ }^{1}$ Instituto Nacional de Investigaciones Forestales Agropecuarias y Pecuarias-Conjunto SEDAGRO. \\ Metepec, Estado de México. CP. 52140. Tel. 722 2329833. (ayaladany84@hotmail.com; \\ druizsaenz@hotmail.com; lopez.humberto@inifap.gob.mx). ${ }^{2}$ Centro Nacional de Recursos Genéticos- \\ INIFAP. Tepatitlán de Morelos, Jalisco, México. CP. 47600. Tel. 378 1065020, ext. 5102. \\ (cruz.esmeralda@inifap.gob.mx). \\ ${ }^{\S}$ Autor para correspondencia: lopez.humberto@inifap.gob.mx.
}

\section{Resumen}

Un problema común en los procesos criogénicos es la supervivencia a temperatura ultra baja (-196 $\left.{ }^{\circ} \mathrm{C}\right)$. Con base a los efectos fisiológicos del ácido salicílico (AS) en la señalización de respuestas de tolerancia a estrés, el objetivo fue evaluar el efecto de AS en el incremento de la supervivencia y en el crecimiento de yemas de papa sometidas a criogenia. Microplantas del clon avanzado 0627 del Banco de Germoplasma in vitro del Programa de Papa en Metepec Estado de México, México, fueron incubadas por 28 días en AS $\left(0,10^{-6}\right.$ y $\left.10^{-5} \mathrm{M}\right)$ y sometidas posteriormente a proceso criogénico. Efecto de AS en regeneración de plantas: se observó un incremento significativo en la supervivencia en plantas pretratadas con AS $10^{-5}$ y $10^{-6} \mathrm{M}$ con respecto al testigo (1.66-2.04 veces respectivamente). El peso fresco, longitud de tallo y raíz incrementaron significativamente en microplantas pretratadas con AS con respecto al testigo. Criogenia: ambas concentraciones de AS $10^{-5}$ y $10^{-6} \mathrm{M}$ indujeron significativamente mayor supervivencia (2.17-3.21 veces respectivamente) de explantes a criogenia. Plantas preincubadas en AS y regeneradas después de ser sometidas $1 \mathrm{~h}$ a criogenia incrementaron significativamente la longitud de raíz. El testigo presentó formación de callo, ausente en las plantas tratadas con AS. La combinación AS-criogenia favoreció el desarrollo de plantas sometidas a métodos que emplean temperaturas ultra bajas en papa con fines de criopreservación de germoplasma o en crioterapia para la obtención de materiales libres de virus.

Palabras claves: papa, regeneración in vitro, ultra baja temperatura.

Recibido: junio de 2019

Aceptado: octubre de 2019 


\section{Introducción}

Un factor importante para la seguridad alimentaria es la adecuada conservación de los recursos genéticos disponibles y la preservación de la diversidad vegetal. La conservación de recursos genéticos puede realizarse por estrategias a corto o largo plazo. Una de estas estrategias a largo plazo es el uso de procedimientos criogénicos, los cuales involucran el almacenamiento de materiales vegetales como lo son maíz (Zea mays) frijol ñame (Sphenostylis stenocarpa), caupí (Vigna unguiculata), maní bambara (Vigna subterranea), soya (Glicine max), palma aceitera (Elaeis guineensis), cacao (Theobroma cacao), coco (Cocos nucifera), aguacate (Persea americana), mango (Mangifera indica) y café (Coffea spp.) entre otras especies (Sánchez-Chiang y Jiménez, 2010).

Este almacenamiento se realiza a temperaturas ultra bajas $\left(-196{ }^{\circ} \mathrm{C}\right)$ utilizando nitrógeno líquido (NL) (Day et al., 2008; Hamilton et al., 2009). A esta temperatura todas las funciones celulares se suspenden y por lo tanto el tejido puede ser almacenado sin cambios o deterioro por largos periodos (Kaczmarczyk et al., 2012). En Solanum tuberosum se han probado exitosamente diversos métodos criogénicos, como encapsulación-deshidratación, vitrificación y D-crioplaca (Hao et al., 2002; Halmagyi et al., 2005; Hirai, 2011; Yamamoto et al., 2015). Wang et al. (2006), propusieron el uso de métodos criogénicos para la conservación a largo plazo de accesiones de papa y para la eliminación de virus.

Sin embargo, la respuesta fisiológica a la criogenia estará en función del genotipo, siendo necesaria la optimización de los métodos para incrementar la sobrevivencia (Rivera et al., 2008). Durante criogenia los tejidos celulares son susceptibles a diversos tipos de estrés como, mecánico, osmótico y temperatura, los cuales conducen a un estrés oxidativo. (Baek y Skinner, 2012). Exposiciones a temperaturas extremas como frio $\left(0-20^{\circ} \mathrm{C}\right)$ o congelamiento $\left(<0{ }^{\circ} \mathrm{C}\right)$ resultan en la disminución de la sobrevivencia (Chinnusamy et al., 2007), produciendo daño oxidativo (Horváth et al., 2007).

La formación de especies reactivas de oxígeno (EROS) en criogenia puede ocurrir durante varios de los pasos involucrados, (Kaczmarczyk et al., 2012). Se ha demostrado que la adición de antioxidantes exógenos como: ácido ascórbico, vitamina $\mathrm{E}$ y glutatión en zarzamora y cítricos durante la crioconservación, resultó en una mayor supervivencia (Wang and Deng, 2004; Uchendu et al., 2010). Las plantas poseen sistemas antioxidantes enzimáticos y no enzimáticos para resistir el estrés, enzimáticos como catalasa (CAT), peroxidasa (POX), súper óxido dismutasa (SOD) y no enzimáticos como el ácido ascórbico, glutatión y $\alpha$ tocoferol (Gill and Tuteja, 2010).

Se ha reportado que el ácido salicílico participa en la señalización de la actividad antioxidante en papa bajo temperaturas extremas (Mora-Herrera et al., 2005; Aguilar-Camacho et al., 2016). El ácido salicílico (AS) induce un incremento de enzimas antioxidantes durante periodos de preaclimatación a bajas temperaturas en plantas tratadas (Sasheva et al., 2010; López-Delgado et al., 2018). Mora-Herrera y López-Delgado (2006), encontraron que el AS induce tolerancia a temperatura de congelación $\left(-6^{\circ} \mathrm{C}\right)$ en papa. El AS también ha sido probado en criogenia en diversas especies, induciendo tolerancia al estrés por congelamiento (Wang and Valkonen, 2009b;

Li et al., 2011; Pathirana et al., 2016). 
En ejes embrionarios de Persian lilac (Melia azedarach L.) reportaron que el AS incrementó significativamente los porcentajes de viabilidad después de ser sometidos a crioconservación (Bernard et al., 2002). El objetivo de este trabajo fue probar el efecto potencial del AS en el incremento de la supervivencia y respuestas de crecimiento en yemas de papa sometidas a proceso criogénico.

\section{Materiales y métodos}

Plantas de Solanum tuberosum L. del clon avanzado 06-27 con tolerancia a tizón tardío del Banco de Germoplasma in vitro del Laboratorio de Fisiología y Biotecnología de papa del INIFAP en Metepec Estado de México, México, fueron seleccionadas con base en su baja tolerancia a criogenia observada en trabajos preliminares del laboratorio. Las plantas fueron incubadas por 28 días en medio de propagación MS (Murashige y Skoog, 1962) bajo tratamiento de AS, 0, $10^{-6}$ y $10^{-5} \mathrm{M}$ (Mora-Herrera et al., 2005; Aguilar-Camacho et al., 2016). Yemas nodales (1-2.5 mm) de dichas microplantas se evaluaron bajo las siguientes condiciones.

a) efecto de $\mathrm{AS}$ en la regeneración de plantas posterior al tratamiento: veinte yemas se subcultivaron a medio MS sin AS y se incubaron por 15 días, para evaluar supervivencia, peso fresco, altura de la planta y longitud de raíz. La supervivencia se evaluó considerando oxidación y turgencia.

b) criogenia: veinte yemas se subcultivaron a medio MS sin AS y se incubaron por 3 días, para ser sometidas a criogenia. El método criogénico utilizado fue Deshidratación-crioplaca (Dcrioplaca) (Yamamoto et al., 2015), modificado por Arizaga et al. (2017) el cual consiste en los siguientes pasos.

1) un volumen de $2 \mu \mathrm{L}$ de alginato de sodio ( $2 \% \mathrm{p} / \mathrm{v}$ alginato de sodio/0.4 $\mathrm{M}$ de sacarosa en una solución basal de medio MS), se colocó en cada uno de los 10 pozos de una crioplaca (7 x 37 x $5 \mathrm{~mm})$.

2) cada una de las yemas fueron transferidas individualmente a cada uno de los pozos de la crioplaca, para posteriormente cubrirlas con la solución de alginato de sodio.

3) la crioplaca con las yemas se cubrió con una hoja estéril de papel BEMCOT (7 x 30 mm).

4) se agregó $1 \mathrm{~mL}$ de solución de cloruro de calcio ( $0.1 \mathrm{M}$ de cloruro de calcio/0.4 M de sacarosa en solución basal de MS) hasta cubrir la crioplaca. La polimerización del alginato de sodio/cloruro de calcio se completó después de 15 min a temperatura ambiente, eliminando el exceso de solución de cloruro de calcio.

5) la crioplaca con las yemas y el papel adherido se transfirieron a una solución de carga (SC) (2 M de glicerol/1 M de sacarosa en solución basal de MS), durante 45 min, se eliminó el exceso de SC.

6) la crioplaca con las yemas y el papel adherido se transfirieron a una caja petri con $35 \mathrm{~g}$ de sílica gel para el proceso de deshidratación, durante 90 min a $24{ }^{\circ} \mathrm{C}$.

7) después de la deshidratación, las crioplacas se transfirieron a criotubos y se sumergieron directamente en nitrógeno líquido (NL) durante $60 \mathrm{~min}$.

8) las crioplacas fueron retiradas del NL y se transfirieron a criotubos con $2 \mathrm{~mL}$ de solución de sacarosa (1 M en medio basal MS) durante 15 min a temperatura ambiente.

9) Las yemas unidas al papel Bemcot se retiraron de la crioplaca y se colocaron en cajas petri con medio MS durante $24 \mathrm{~h}$. 
10) posteriormente se eliminó la capsula de alginato de sodio dejando expuesta la yema, la cual se subcultivo a medio MS fresco.

11) 15 días posteriores al subcultivo, se evaluó la supervivencia de las plantas regeneradas, considerando vivas aquellas que reanudaron su crecimiento; así como la formación de callo y longitud de raíz $(n=8-20)$.

El análisis estadístico se realizó por medio de análisis de varianza (Anova) y prueba de Duncan (Duncan, 1955) en todos los experimentos, mediante el programa Statgraphics Centurion XVI. El nivel de confiabilidad establecido fue de $p<0.05$. Los experimentos se realizaron por triplicado.

\section{Resultados y discusión}

\section{Efectos del AS en la regeneración de plantas posterior al tratamiento}

Después del tratamiento de AS en las microplantas tratadas con AS $10^{-6}$ y $10^{-5} \mathrm{M}$, se observaron respuestas fisiológicas como mayor supervivencia, longitud de raíz y altura de la planta. Un incremento significativo en la supervivencia de 2.04 y 1.66 veces respectivamente respecto al testigo (Figura 1).

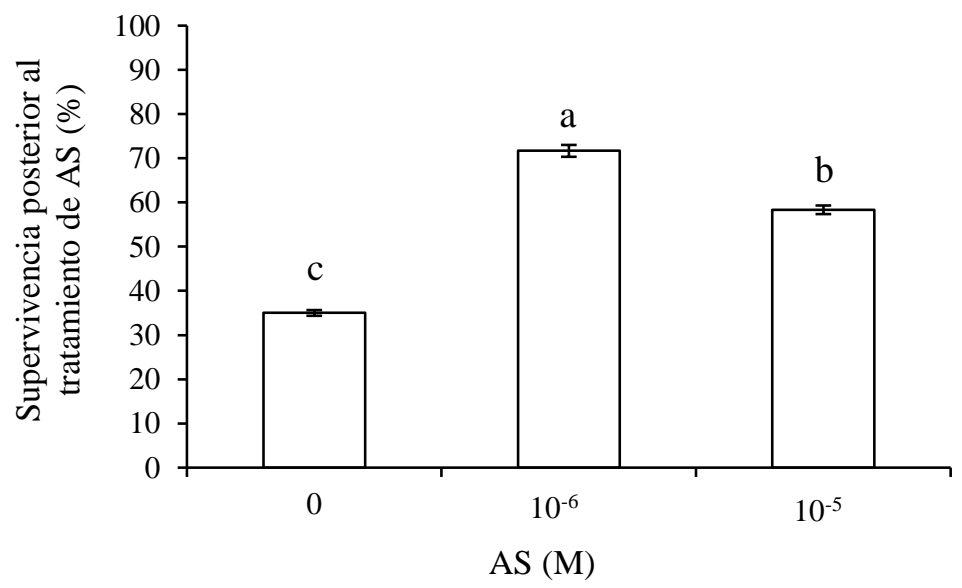

Figura 1. Efecto del AS en la supervivencia del clon de papa 06-27 posterior al tratamiento. Variables evaluadas 15 días posteriores al subcultivo. Datos obtenidos de 3 experimentos $(n=20)$. Datos analizados con Anova y prueba de Duncan $(p<0.05)$. Letras diferentes indican diferencias significativas respecto al testigo.

Efectos similares en el incremento de supervivencia por AS se han encontrado en diversas especies al utilizarlo en diferentes concentraciones, como en Astragalus adsurgens (1.64 veces) (Luo et al., 2001) y Coffea arabica (1.57 veces) (Quiroz-Figueroa et al., 2001).

Las microplantas pretratadas con AS $10^{-6}$ y $10^{-5} \mathrm{M}$ mostraron un incremento significativo en la longitud de raíz de 3.28 y 2.87 veces respectivamente, así como en la altura de la planta de 2.6 y 1.87 veces respecto al testigo (Figuras $2 \mathrm{a}$ y $2 \mathrm{~b}$ ). 

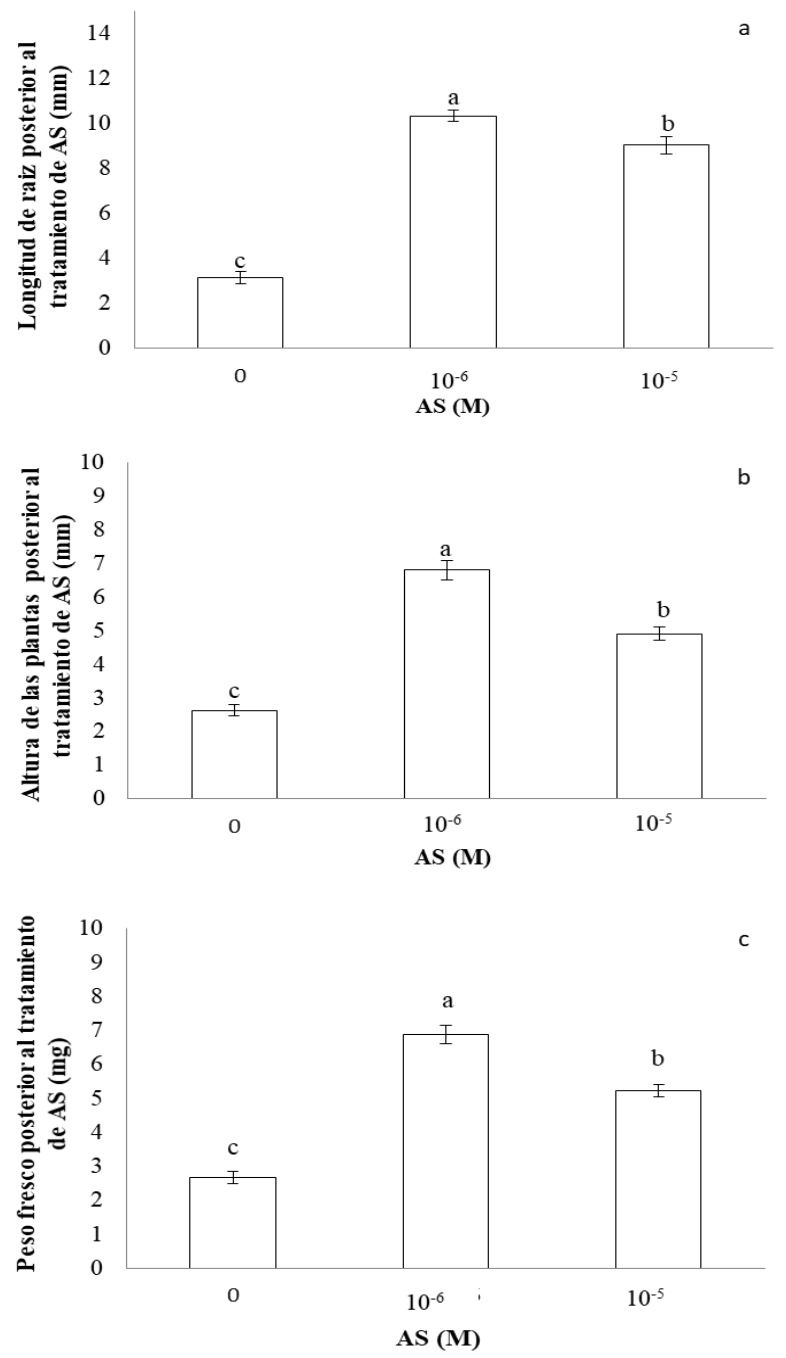

Figura 2. Efecto del AS en el crecimiento del clon de papa 06-27 posterior al tratamiento. a) longitud de raíz; b) altura de la planta; y c) peso fresco. Variables evaluadas 15 días posteriores al subcultivo, datos obtenidos de 3 experimentos $(n=20)$ y analizados con Anova y prueba de Duncan $(p<0.05)$. Letras diferentes indican diferencias significativas respecto al testigo.

Los resultados obtenidos en esta investigación son de alto interés, debido a que un mayor desarrollo de la raíz es importante para el establecimiento tanto en condiciones in vitro como ex vitro de cualquier especie, debido a que está directamente relacionado con la absorción de nutrientes y a su vez induce incremento en el crecimiento (Ruiz, 2000; Selles et al., 2003; Callejas-Rodríguez et al., 2012).

Lo anterior coincide con lo reportado por Larqué-Saavedra y Martín-Mex (2007), ya que encontraron un incremento de densidad y longitud de raíz de plantas tratadas con AS. De acuerdo a Sakhabutdinova et al. (2003), tratamientos de AS pueden incrementar la división celular en las raíces de las plántulas aumentando su crecimiento general, lo anterior puede estar relacionado con el efecto del AS sobre el incremento de la longitud de raíz observado en este trabajo en microplantas de papa (Figura 2a). 
Otros trabajos que concuerdan con los resultados obtenidos en esta investigación correspondientes a los efectos de AS en longitud de raíz y altura de la planta (Figura 2a, 2b), se han reportado en diferentes cultivos tales como Hibiscus (variedades acetocela y moscheutos) (Sakhanokho and Kelley, 2009), maíz (Gunes et al., 2007), soya (Gutiérrez-Coronado et al., 1998) y trigo (Shakirova et al., 2003). Microplantas pretratadas con AS $10^{-6}$ y $10^{-5} \mathrm{M}$ mostraron un incremento significativo en peso fresco de 2.58 y 1.96 veces respectivamente, en comparación con el testigo (Figura 2c). Páez-García et al. (2015), mencionan que la aplicación de AS induce un incremento de la biomasa debido a un mayor desarrollo en el sistema radical, lo que conlleva a una mejor absorción de agua y nutrientes.

\section{Criogenia}

Las microplantas que reanudaron su crecimiento proveniente de los tratamientos de AS $10^{-6}$ y $10^{-5}$ $\mathrm{M}$ mostraron un incremento en la supervivencia a criogenia de 3.21 y 2.17 veces respectivamente, respecto al testigo (Figura 3).

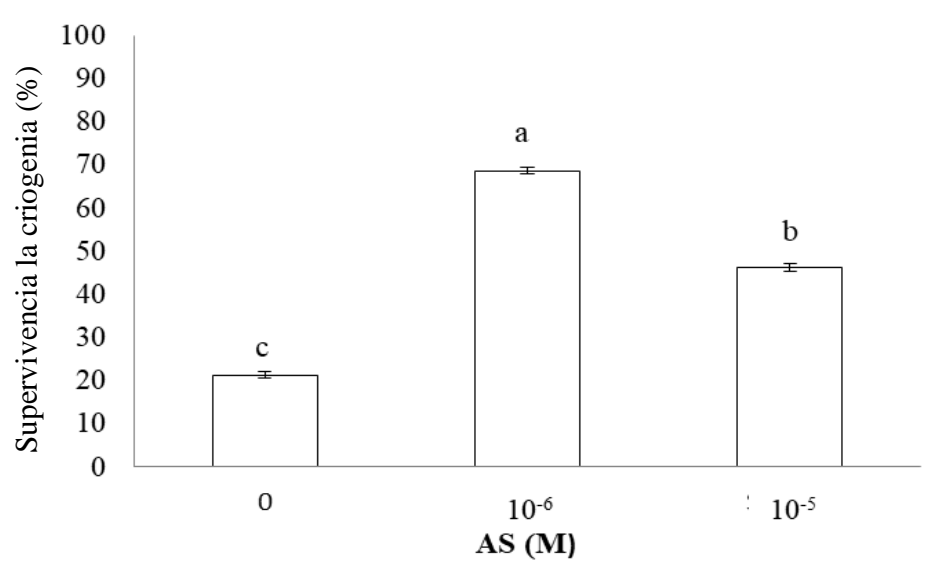

Figura 3. Efecto del AS en la supervivencia del clon de papa 06-27 después de criogenia. Variables evaluadas 15 días posteriores al subcultivo. Datos obtenidos de 3 experimentos $(n=8-20)$ y analizados con Anova y prueba de Duncan $(p<0.05)$. Letras diferentes indican diferencias significativas respecto al testigo.

Dichos resultados contrastan con los valores obtenidos por Rivera et al. (2008), quien obtuvo hasta $52 \%$ de sobrevivencia en ausencia de pretratamientos de AS. Los resultados observados pueden relacionarse con una reducción del estrés oxidativo por efecto de AS como ha sido demostrado en papa bajo diferentes tipos de estrés, como mayor supervivencia bajo termoterapia (hasta 2 veces) (Aguilar-Camacho et al., 2016).

Mayor peso de tubérculo en plantas infectadas con fitoplasma (1.88 veces) (Sánchez-Rojo et al., 2011), especialmente mayor supervivencia a temperaturas bajas (1.77-2.35 veces) (Mora-Herrera et al., 2005; López-Delgado et al., 2018). Después del proceso criogénico se observó ausencia de callo en las microplantas provenientes de ambos tratamientos de AS mientras que el testigo presentó un $68 \%$ de formación de callo sin la formación de brote de tallo (Figura 4). 


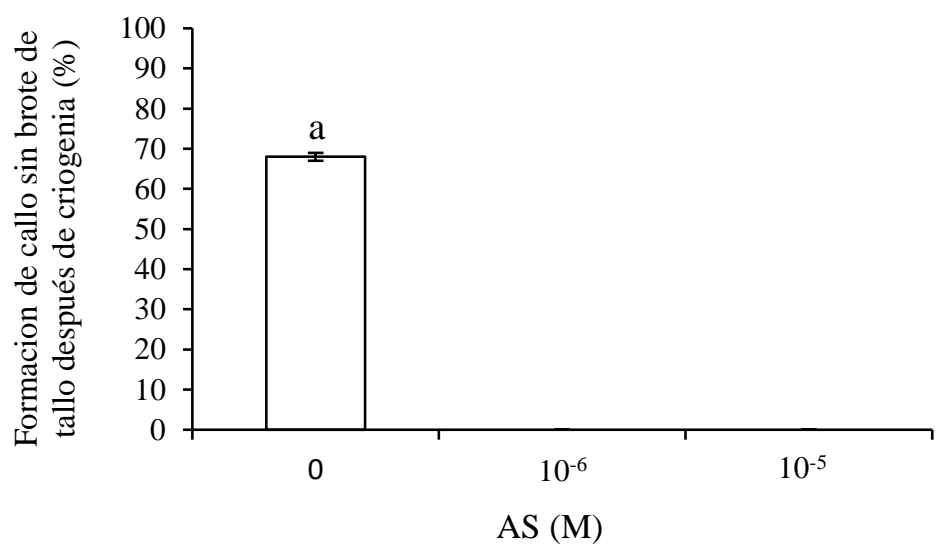

Figura 4. Efecto del AS en el desarrollo del clon de papa 06-27 después de criogenia. Formación de callo sin desarrollo de brote de tallo. Evaluaciones realizadas 15 días posteriores al proceso criogénico. Datos obtenidos de 3 experimentos $(n=8-20)$. Datos analizados con Anova y prueba de Duncan $(p<0.05)$. Letras diferentes indican diferencias significativas respecto al testigo.

Durante la regeneración de plantas después de las técnicas criogénicas, es muy deseable una regeneración directa sin la formación de callo, debido que asegura la estabilidad genética de las plantas obtenidas (Reed, 2008). Los resultados en esta investigación demuestran el potencial del AS para incrementar la supervivencia en criogenia en genotipos de papa que presentan baja supervivencia en dichos procesos además de prevenir la formación de callo en la regeneración, ya que se reduce el riesgo de variación somaclonal, favoreciendo la conservación de las características fenotípicas y genotípicas del material vegetal.

Aunado a la ausencia de callo en los explantes regenerados, se obtuvo un incremento significativo en la longitud de raíz en plantas pretratadas con AS $10^{-6}$ y $10^{-5} \mathrm{M}$ de 3.35 y 2.36 veces respectivamente después de criogenia (Figura 5).

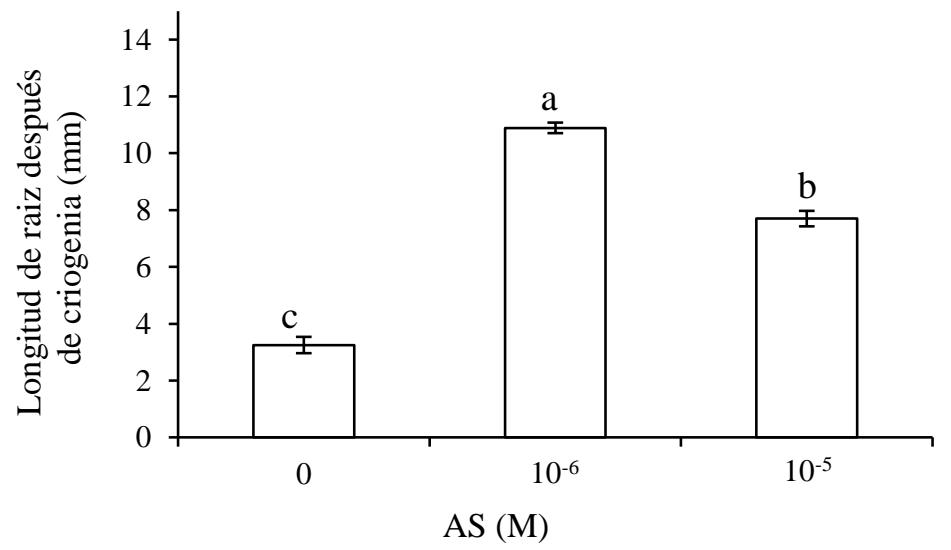

Figura 5. Efecto del AS en el desarrollo del clon de papa 06-27 después de criogenia. Formación de raíz. Evaluaciones realizadas 15 días posteriores al proceso criogénico, con datos obtenidos de 3 experimentos $(\mathrm{n}=8-20)$ y analizados con Anova y prueba de Duncan $(p<0.05)$. Letras diferentes indican diferencias significativas respecto al testigo. 
En comparación con el testigo, el éxito de una óptima regeneración después de la exposición a nitrógeno líquido se basa en un estado fisiológico y morfológico adecuado del explante que fue sometido al método criogénico (Engelmann et al., 2008).

Pretratamientos con AS pueden ser una importante herramienta para una mejor supervivencia a técnicas de criopreservación o crioterapia en papa, en genotipos con baja supervivencia a temperaturas ultra bajas ya que es una molécula señalizadora de inducción de tolerancia al estrés y reguladora del crecimiento en plantas (Horváth, 2007), además que interviene en el desarrollo de raíces en condiciones de estrés por bajas temperaturas (Huang y Villanueva, 1993; Melkonian et al., 2004).

\section{Conclusiones}

En esta investigación se observó el efecto del AS como inductor de tolerancia a temperaturas ultra bajas empleadas en procesos criogénicos en plantas de papa que presentan baja supervivencia a criogenia, aumentando los porcentajes de supervivencia.

Aunado a ello también existe un efecto promotor del AS en el desarrollo de los explantes sometidos al proceso criogénico ya que se obtuvo un incremento significativo en el desarrollo de las raíces de dichos explantes después de su regeneración. AS evitó la formación de callo lo cual es un factor importante para mantener la estabilidad genética en cultivos como Solanum tuberosum.

\section{Literatura citada}

Aguilar-Camacho, M.; Mora-Herrera, M. E. and López-Delgado, H. A. 2016. Potato virus X (PVX) elimination as short and long term effects of hydrogen peroxide and salicylic acid is differentially mediated by oxidative stress in synergism with thermotherapy. Am. J. Potato Res. 93(4):360-367.

Arizaga, M. V.; Navarro, O. F. V.; Martínez, C. R. C.; Gutiérrez, E. J. C.; Delgado, H. A. L.; Yamamoto, S. I.; Watanabe, K. and Niino, T. 2017. Improvement to the D cryo-plate protocol applied to practical cryopreservation of in vitro grown potato shoot tips. The Hortic. J. 86(2):222-228.

Baek, K. H. and Skinner, D. Z. 2012. Production of reactive oxygen species by freezing stress and the protective roles of antioxidant enzymes in plants. Journal of Agricultural Chemistry and Environment. 1(01):34-40.

Bernard, F.; Shaker-Bazarnov, H. and Kaviani, B. 2002. Effects of salicylic acid on cold preservation and cryopreservation of encapsulated embryonic axes of Persian lilac (Melia azedarach L.). Euphytica. 123(1):85-88.

Callejas-Rodríguez, R.; Rojo-Torres, E.; Benavidez-Zabala, C. y Kania-Kuhl, E. 2012. Crecimiento y distribución de raíces y su relación con el potencial productivo de parrales de vides de mesa. Agrociencia. 46(1):23-35.

Chinnusamy, V.; Zhu, J. and Zhu, J. K. 2007. Cold stress regulation of gene expression in plants. Trends in Plant Sci. 12(10):444-451.

Day, J. G.; Harding, K. C.; Nadarajan, J. and Benson, E. E. 2008. Cryopreservation: conservation of bioresources at ultra low temperatures. In: molecular biomethods handbook. Humana Press. 52(2):917-947. 
Duncan, D. B. 1955. Multiple range and multiple F tests. Biometrics. 11(1):1-42.

Engelmann, F.; Arnao, M. T. G.; Wu, Y. and Escobar, R. 2008. The development of encapsulation dehydration. In: plant cryopreservation: A practical guide. Springer. 4(1):59-75.

Gill, S. S. and Tuteja, N. 2010. Reactive oxygen species and antioxidant machinery in abiotic stress tolerance in crop plants. Plant Physiol. Biochem. 48(12):909-930.

Gunes, A.; Inal, A.; Alpaslan, M.; Eraslan, F.; Bagci, E. G. and Cicek, N. 2007. Salicylic acid induced changes on some physiological parameters symptomatic for oxidative stress and mineral nutrition in maize (Zea mays L.) grown under salinity. J. Plant Physiol. 164(6):728-736.

Gutiérrez-Coronado, M. A.; Trejo-López, C. and Larqué-Saavedra, A. 1998. Effects of salicylic acid on the growth of roots and shoots in soybean. Plant Physiol. Biochem. 36(8):563-565.

Halmagyi, A.; Deliu, C. and Coste, A. 2005. Plant regrowth from potato shoot tips cryopreserved by a combined vitrification-droplet method. CryoLetters. 26(5):313-322.

Hamilton, K. N.; Ashmore, S. E. and Pritchard, H. W. 2009. Thermal analysis and cryopreservation of seeds of Australian wild Citrus species (Rutaceae): Citrus australasica, C. inodora and C. garrawayi. CryoLetters. 30(4):268-279.

Hao, Y. J.; You, C. X. and Deng, X. X. 2002. Analysis of ploidy and the patterns of amplified fragment length polymorphism and methylation sensitive amplified polymorphism in strawberry plants recovered from cryopreservation. CryoLetters. 23(1):37-46.

Hirai, D. 2011. Gelled droplet vitrification improves recovery of cryopreserved potato germplasm. CryoLetters. 32(4): 287-296.

Horváth, E.; Szalai, G. and Janda, T. 2007. Induction of abiotic stress tolerance by salicylic acid signaling. J. Plant Growth Reg. 26(3):290-300.

Huang, H. and Villanueva, V. R. 1993. Amino acids, polyamines and proteins during seed germination of two species of Dipterocarpaceae. Springer. Trees Structure and Function. 7(3):189-193.

Kaczmarczyk, A.; Funnekotter, B.; Menon, A.; Phang, P. Y.; Al-Hanbali, A.; Bunn, E. and Mancera, R. L. 2012. Current issues in plant cryopreservation. In: current frontiers in cryobiology. InTech. 14(1):418-438.

Larqué-Saavedra, A. and Martin-Mex, R. 2007. Effects of salicylic acid on the bioproductivity of plants. In Salicylic acid: A Plant Hormone. Springer. 2(1):15-23.

Li, Y.; Liu, C.; Li, T.; Wang, C.; Xiao, Y.; Zhang, L.; Jin, D.; Zhao, Y.; Wang. Z.; Cao, J. and Hao, L. 2011. Regulatory role of exogenous salicylic acid in the response of Zoysia japonica plants to freezing temperatures: a comparison with cold-acclimatisation. The J. Hortic. Sci. Biotechnol. 86(3):277-283.

López-Delgado, H. A.; Martínez-Gutiérrez, R.; Mora-Herrera, M. E. and Torres-Valdés, Y. 2018. Induction of freezing tolerance by the application of hydrogen peroxide and salicylic acid as tuber-dip or canopy spraying in Solanum tuberosum L. Plants. Potato Res. 61(3):195-206.

Luo, J. P.; Jiang, S. T. and Pan, L. J. 2001. Enhanced somatic embryogenesis by salicylic acid of Astragalus adsurgens Pall: relationship with $\mathrm{H}_{2} \mathrm{O}_{2}$ production and $\mathrm{H}_{2} \mathrm{O}_{2}$-metabolizing enzyme activities. Plant Science. 161(1):125-132.

Melkonian, J.; Yu, L. X. and Setter, T. L. 2004. Chilling responses of maize (Zea mays L.) seedlings: root hydraulic conductance, abscisic acid, and stomatal conductance. J. Exp. Bot. 55(403):1751-1760. 
Mora Herrera, M. E. y López Delgado, H. A. 2006. Tolerancia a baja temperatura inducida por ácido salicílico y peróxido de hidrógeno en microplantas de papa. Rev. Fitotec. Mex. 29(2):81-85.

Mora-Herrera, M. E.; López-Delgado, H.; Castillo-Morales, A. and Foyer, C. H. 2005. Salicylic acid and $\mathrm{H}_{2} \mathrm{O}_{2}$ function by independent pathways in the induction of freezing tolerance in potato. Physiol. Plantarum. 125(4):430-440.

Murashige, T. and Skoog, F. 1962. A revised medium for rapid growth and bioassays with tobacco tissue cultures. Physiol. Plantarum. 15(3):473-497.

Paez-García, A.; Motes, C.; Scheible, W. R.; Chen, R.; Blancaflor, E. and Monteros, M. 2015. Root traits and phenotyping strategies for plant improvement. J. Plants. 4(2):334-355.

Pathirana, R.; McLachlan, A.; Hedderley, D.; Panis, B. and Carimi, F. 2016. Pre-treatment with salicylic acid improves plant regeneration after cryopreservation of grapevine (Vitis spp.) by droplet vitrification. Acta Physiologiae Plantarum. 38(12):1-11.

Quiroz-Figueroa, F.; Méndez-Zeel, M.; Larqué-Saavedra, A. and Loyola-Vargas, V. 2001. Picomolar concentrations of salicylates induce cellular growth and enhance somatic embryogenesis in Coffea arabica tissue culture. Plant Cell Reports. 20(8):679-684.

Reed, B. M. 2008. Cryopreservation: Practical Considerations. In: plant cryopreservation: a practical guide. Springer. 1(1):3-13.

Rivera, Á.; Valbuena, R.; Hidalgo, R. y Moreno, J. 2008. Crioconservación de yemas de microtubérculos de papa Solanum tuberosum ssp. andigena mediante desecado de tejidos. Corpoica. Ciencia y Tecnología Agropecuaria. 9(2):37-44.

Ruiz, S. 2000. Dinámica nutricional en cinco parrones de diferente productividad del valle central regado de Chile. Agric. Téc. 60(4):379-398.

Sakhabutdinova, A. R.; Fatkhutdinova, D. R.; Bezrukova, M. V. and Shakirova, F. M. 2003. Salicylic acid prevents the damaging action of stress factors on wheat plants. Bulgarian $\mathrm{J}$. Plant Physiol. 21(1):314-319.

Sakhanokho, H. F. and Kelley, R. Y. 2009. Influence of salicylic acid on in vitro propagation and salt tolerance in Hibiscus acetosella and Hibiscus moscheutos (cv 'Luna Red'). Afr. J. Biotechnol. 8(8):1474-1481.

Sánchez-Chiang, N. y Jiménez, V. M. 2010. Técnicas de conservación in vitro para el establecimiento de bancos de germoplasma en cultivos tropicales. Agron. Mesoam. 21(1):193-205.

Sánchez-Rojo, S.; López-Delgado, H. A.; Mora-Herrera, M. E.; Almeyda-León, H. I.; ZavaletaMancera, H. A. and Espinosa-Victoria, D. 2011. Salicylic acid protects potato plants-from phytoplasma-associated stress and improves tuber photosynthate assimilation. Am. J. Potato Res. 88(2):175-183.

Sasheva, P.; Szalai, G.; Janda, T. and Popova, L. 2010. Study of the behaviour of antioxidant enzymes in the response to hardening and freezing stress in two wheats (Triticum aestivum L.) varieties. Acta Agron. Hungarica. 61(2):161-172

Sellés, G. V. S.; Ferreyra, R. E.; Contreras, G. W.; Ahumada, R. B.; Valenzuela, J. B. and Bravo, R. V. 2003. Drip irrigation management in table grapes cv. Thompson seedless grown on fine textured soils. Agric. Téc. 63(2):180-192.

Shakirova, F. M.; Sakhabutdinova, A. R.; Bezrukova, M. V.; Fatkhutdinova, R. A. and Fatkhutdinova, D. R. 2003. Changes in the hormonal status of wheat seedlings induced by salicylic acid and salinity. Plant Sci. 164(3):317-322. 
Uchendu, E. E.; Muminova, M.; Gupta, S. and Reed, B. M. 2010. Antioxidant and anti-stress compounds improve regrowth of cryopreserved Rubus shoot tips. In Vitro Cellular Developm. Biol. Plant. 46(4):386-393.

Wang, Q. and Valkonen, J. P. 2009b. Cryotherapy of shoot tips: novel pathogen eradication method. Trends Plant Sci. 14(3):119-122.

Wang, Q.; Liu, Y.; Xie, Y. and You, M. 2006. Cryotherapy of potato shoot tips for efficient elimination of potato leafroll virus (PLRV) and potato virus Y (PVY). Potato Res. 49(2):119-129.

Wang, Z. C. and Deng, X. X. 2004. Cryopreservation of shoot-tips of citrus using vitrification: effect of reduced form of glutathione. CryoLetters. 25(1):43-50.

Yamamoto, S. I.; Rafique, T.; Arizaga, M. V.; Fukui, K.; Gutiérrez, E. J. C.; Martínez, C. R. C. and Niino, T. 2015. The aluminum cryo-plate increases efficiency of cryopreservation protocols for potato shoot tips. Am. J. Potato Res. 92(2):250-257. 